\title{
Novel likely pathogenic variants in TMEM126A identified in non-syndromic autosomal recessive optic atrophy: two case reports
}

Katja Kloth ${ }^{1}$, Matthis Synofzik ${ }^{2,3}$, Christoph Kernstock ${ }^{4}$, Simone Schimpf-Linzenbold ${ }^{5}$, Frank Schuettauf ${ }^{6}$, Axel Neu', Bernd Wissinger ${ }^{4}$ and Nicole Weisschuh ${ }^{4^{*}}$ (D)

\begin{abstract}
Background: Reports on autosomal recessive optic atrophy (arOA) are sparse and so far, only one gene has been specifically associated with non-syndromic arOA, namely TMEM126A. To date, all reports of pathogenic TMEM126A variants are from affected individuals of Maghrebian origin, who all carry an identical nonsense variant. Here we report two novel variants in the TMEM126A gene from non-Maghreb individuals, both found in affected individuals with an arOA phenotype.

Case presentation: We report three affected individuals from two families. The proband of family A, a 24-year-old Turkish woman, was diagnosed with visual loss in early childhood but a diagnosis of optic atrophy was only made at 14 years. A diagnostic gene panel revealed a splice donor variant $(c .86+2 \mathrm{~T}>\mathrm{C})$ in homozygous state in the TMEM126A gene. Analysis of this variant based on RNA from whole blood revealed a single aberrant transcript lacking exon 2, presumably representing a functional null allele. Two siblings from family B, a 16-year old Iraqi girl and her 14-year old brother, were diagnosed with optic atrophy in early childhood. A missense variant p.(S36 L) in the TMEM126A gene was identified in homozygous state in a gene panel-based diagnostic setting in both siblings. This missense variant is ultra rare in the general population, affects a highly evolutionarily conserved amino acid and segregates with the disease within the family. The three probands reported in this study had a relatively mild clinical course without any evidence of a syndromic (e.g. neurological) comorbidity, which is in line with previous studies.
\end{abstract}

Conclusions: We provide additional evidence for the implication of biallelic pathogenic TMEM126A variants in arOA. Our findings extend both the mutational spectrum and geographic presence of TMEM126A in arOA. Screening of the entire gene should be considered in affected individuals presenting with features resembling arOA and also from non-Maghrebian descent.

Keywords: Optic atrophy, Autosomal recessive, TMEM126A

\footnotetext{
* Correspondence: nicole.weisschuh@uni-tuebingen.de

${ }^{4}$ Center for Ophthalmology, Institute for Ophthalmic Research, University of

Tübingen, Tübingen, Germany

Full list of author information is available at the end of the article
}

(c) The Author(s). 2019 Open Access This article is distributed under the terms of the Creative Commons Attribution 4.0 International License (http://creativecommons.org/licenses/by/4.0/), which permits unrestricted use, distribution, and reproduction in any medium, provided you give appropriate credit to the original author(s) and the source, provide a link to the Creative Commons license, and indicate if changes were made. The Creative Commons Public Domain Dedication waiver (http://creativecommons.org/publicdomain/zero/1.0/) applies to the data made available in this article, unless otherwise stated. 


\section{Background}

Inherited optic neuropathies are rare ocular diseases that are characterized by early-onset, slowly progressive and bilateral vision loss, central scotoma and color vision disturbances due to dysfunction and degeneration of retinal ganglion cells and the optic nerve [1]. Most of the published genes and pathogenic variants have been associated with autosomal-dominant optic atrophy (adOA, MIM 165500) or maternally inherited Leber hereditary optic neuropathy (LHON, MIM 535000). To this day, only one gene has been specifically associated with non-syndromic autosomal-recessive atrophy (arOA), namely TMEM126A [2]. This gene has been mapped to chromosome 11q14.1, covers a genomic region of 8.5 kB (GenBank NM_032273) and is composed of five exons. So far, all affected individuals reported in the literature originate from the same region, the Maghreb, and are homozygous for a recurrent nonsense variant (p.R55*) in the TMEM126A gene [2-4].

There is an intimate relationship between optic atrophy disease genes and mitochondrial function, since virtually all publicly known disease genes encode proteins that are localized in mitochondria and/or play a role in mitochondrial homeostasis. The exact function of the TMEM126A protein remains unknown. However, extensive studies performed by Hanein and coworkers have shown that it is a protein of the mitochondrial inner membrane with its $\mathrm{N}$ - and C-termini facing the mitochondrial matrix [5]. While affected individuals with pathogenic variants in mitochondrial DNA often clinically present with a multi-systemic disorder, pathogenic variants in nuclear-encoded mitochondrial proteins may be associated with single-system dysfunctions such as adOA caused by pathogenic variants in OPA1, a nuclear encoded mitochondrial large GTPase of the dynamin family that plays a central role in mitochondrial dynamics and cristae junction maintenance [6-8]. Progressive visual loss is the most common symptom in these affected individuals resulting from a dysfunction and eventually loss of retinal ganglion cells and their axons. Recent findings, however, showed that the disease spectrum due to pathogenic OPA1 variants is much broader and includes a variety of syndromic diseases such as adOAplus featuring hearing loss, cerebellar ataxia, ptosis, and peripheral neuropathy or a form of Behr syndrome with additional neuromuscular deficits, partly even with biallelic inheritance $[9,10]$. While individuals with pathogenic variants in TMEM126A have been initially reported to be affected with non-syndromic optic atrophy [2], subsequent studies have shown that some affected individuals also presented with mild extra-ocular symptoms such as auditory neuropathy [3] or sensory-motor axonal neuropathy [4].
In this study, we report three probands from two families harboring two novel putative pathogenic variants in TMEM126A. In-depth neurological examination revealed no syndromic involvement in these affected individuals. Our results emphasize the need for sequencing the entire TMEM126A gene in affected individuals with arOA instead of focusing on the recurrent (p.R55*) variant, and testing also affected individuals outside from Maghreb. Moreover, the identification of a first missense variant may provide a hint on protein domains crucial for the mitochondrial function of TMEM126A.

\section{Case presentation \\ Affected individuals}

Family A was recruited and clinically examined at the Centre for Ophthalmology and at the Department of Neurodegenerative Diseases, University of Tübingen, Germany. Ethical approval (dated October 23, 2017; Project number 637/2017BO1) and informed consent for all individuals was obtained. DNA from whole blood was extracted from all family members using standard protocols. Total RNA was isolated from whole blood drawn in PAXgene tubes using the PAXgene blood RNA Kit (Qiagen, Hilden, Germany).

Family B was recruited and clinically examined at the Center for Ophthalmology and at the Institute of Human Genetics, University Medical Center Hamburg-Eppendorf, Hamburg, Germany. Ethical approval (dated March 1, 2016; Project number PV3802) and informed consent for all individuals including research purposes was obtained. DNA from whole blood was extracted from all family members using standard protocols.

\section{Clinical investigation}

Ophthalmic examination of the affected probands of family A (II:1) and family B (II:1 and II:2) included fundus photography, best corrected visual acuity (BCVA, Snellen), measurement of intraocular pressure, perimetry and spectral domain optical coherence tomography (SD-OCT; Heidelberg Engineering GmbH, Heidelberg, Germany). Details on methodology have already been described $[10,11]$. Neurological examination of the affected probands of family A (II:1) and family B (II:1, II:2) included assessment of cognitive function, primary sensory modalities, reflexes, coordination and gait. Nerve conduction studies were performed in subject A (II:1). Magnetic resonance imaging (MRI) of the head was also performed in proband II:1 of family A and proband II:1 of family B.

\section{Panel sequencing and data filtering}

Sequencing libraries were prepared starting from DNA using the SureSelectXT workflow (Agilent, Santa Clara, $\mathrm{CA}$ ) and a custom-design enrichment kit (CeGaT EYE, 
version 8) for the index patients. Library preparation and capture was performed according to the manufacturer's instructions and paired-end sequencing was performed on a HiSeq4000 instrument (Illumina, San Diego, CA) (for family A) or on a NovaSeq6000 instrument (Illumina, San Diego, CA) (for family B) with $2 \times$ 100 base pairs (bp) read length, yielding $5 \mathrm{Gbp}$ and 5.3 Gbp of data, respectively. After demultiplexing (Illumina bcl2fastq 2.19), adapters were trimmed with Skewer version 0.1.116 [12]. Trimmed raw reads were aligned to the human genome (hg19) with the Burrows-Wheeler Aligner (BWA-mem version 0.7.2) [13]. Reads likely arising from PCR duplication as well as reads that aligned at more than one locus were discarded (CeGaT internal software). Average coverage on target was 522x (99.2\% $>10 x, 99.53 \%>30 x)$ for family A and 1145x $(99.7 \%>10 x$, $99.7 \%>30 x)$ for family B. Sequence variants were called (VarScan 2.4.2, CeGaT extended version) with a minimum variant allele frequency of $5 \%$. Calls resulting from technical artefacts were removed (CeGaT internal software). Resulting variants were annotated with population frequencies from dbSNP (release 149 and 150) [14], ExAC version 0.3.1 [15] (for family A), gnomAD version 2.0.1 [15] (for family B) and an internal database (CeGaT), with functional predictions from dbNSFP (3.0c and 3.4c) [16], with publications from HGMD (16.4 and 17.2) [17], and with transcript information from Ensembl [18], RefSeq [19], and CCDS [20]. Variants were filtered to remove frequent variants (minor allele frequency $1.5 \%$ ). All variants were manually assessed before inclusion in the final report. Analysis of family members (segregation analysis) was performed by standard Sanger sequencing.

\section{CDNA analysis}

Total RNA isolated from whole blood was reverse transcribed using the Transcriptor First Strand cDNA Synthesis Kit (Roche, Mannheim, Germany) according to the manufacturer's recommendations. The cDNA was PCR amplified using a forward primer located in exon 1 of TMEM126A (GTGGCTGAGGAAGGAGGAG) and a reverse primer located in exon 4 (CAGTCCACT CCGTGTTATGG). Sequence analysis of RT-PCR products was performed as described previously [21].

\section{Clinical findings}

The affected proband from family $\mathrm{A}$ is a 24-year-old Turkish woman, born to unaffected parents (Fig. 1, II:1 in the pedigree). Consanguinity was not reported. Family history revealed three healthy sisters. At age 6, the proband noticed a decrease in visual acuity but a diagnosis of optic atrophy was only made at 14 years of age. Fundus examination at 24 years of age revealed pale discs (see Fig. $2 a+b$ ). Visual acuity was reduced to 20/100
(OD) and 20/200 (OS). Optical coherence tomography showed a markedly reduced peripapillary retinal nerve fiber layer in the temporal sector on both eyes (see Fig. $3 a+b)$. Perimetry showed centrocecal relative scotomas consistent with papillary atrophy (see Fig. 4a).

Neurological in-depth examination by a neurological expert with long-standing experience in examining neurogenetic patients (M.S.) revealed no clinical signs of damage to any neurological system, in particular not of the pyramidal, basal ganglia, cerebellar, spinal or peripheral nerve system. Nerve conduction studies showed normal results for sural and tibial nerves. Also her cognitive abilities were within the full normal range, with the proband finishing regular school and working full-time on the regular job market as a cashier in a grocery store.

The affected probands from family B are a 16-year old Iraqi girl and her 14-year old brother, born to unaffected consanguineous parents (Fig. 1, Family B, I:1 and I:2). Family history reported four healthy sisters (II:3-5; II:7) and one healthy brother (II:6). A grandfather on the mother's side was reported to suffer from blindness at the age of 20 years. The girl was initially diagnosed with poor vision and achromatopsia in early childhood. The clinical diagnosis was revised to optic atrophy when visual evoked potentials showed reduced potentials and delayed latency periods at the age of 14 . Fundus examination at age 15 revealed temporal pallor of the optic discs and the macular reflex was diminished (see Fig. $2 \mathrm{c}+\mathrm{d}$ ). Visual acuity at this point was reduced to $20 / 100$ (proximity) and 50/100 (distance). Peripapillary retinal nerve fiber layer showed temporal thinning similar to proband A (see Fig. $3 \mathrm{c}+$ d). Perimetry showed central and paracentral defects. Additionally, arcuated defects were recorded within the temporal visual fields (see Fig. $4 b+c$ ) that could not be attributed to the physiognomy of the proband. The boy was diagnosed during infancy with reduced visual acuity in one eye and was reported to suffer from nyctalopia. Ophthalmological examination revealed slight temporal pallor of the optic discs and the macular reflex was diminished (see Fig. 2e $+\mathrm{f}$ ). Visual acuity at this point was $25 / 100$ (proximity) and $10 / 100$ (distance). Peripapillary retinal nerve fiber layer showed temporal thinning (see Fig. $3 e+f$ ). Perimetry showed paracentral defects (see Fig. $4 d+e$ ).

Neurological in-depth examination of both children by a trained neuropediatrician and a skilled clinical geneticist revealed no signs of neurological disease or dysfunction, in particular not of the pyramidal, basal ganglia, cerebellar, spinal or peripheral nerve system. Their cognitive abilities were within the full normal range, with both affected individuals effectively attending regular schooling. 


\section{Family A - M:c.86+2T>C}

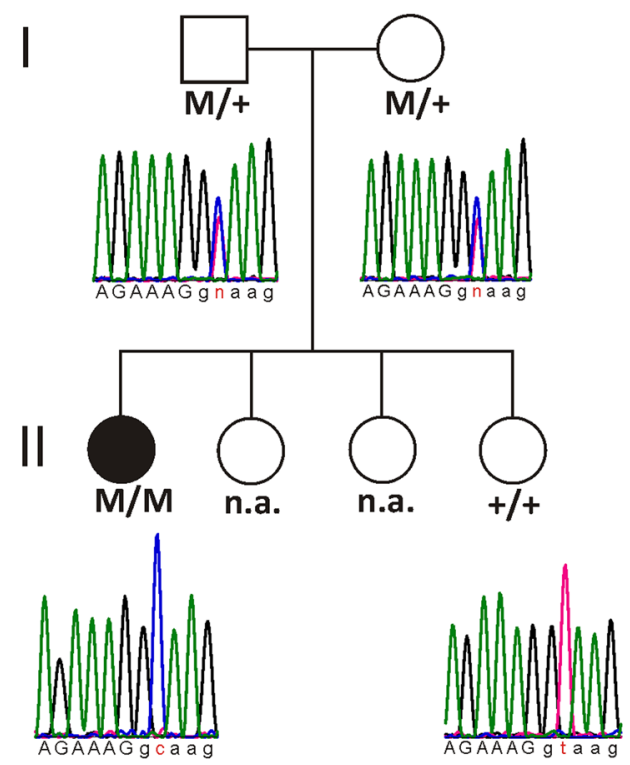

Family B - M:c.107C >T/p.S36L

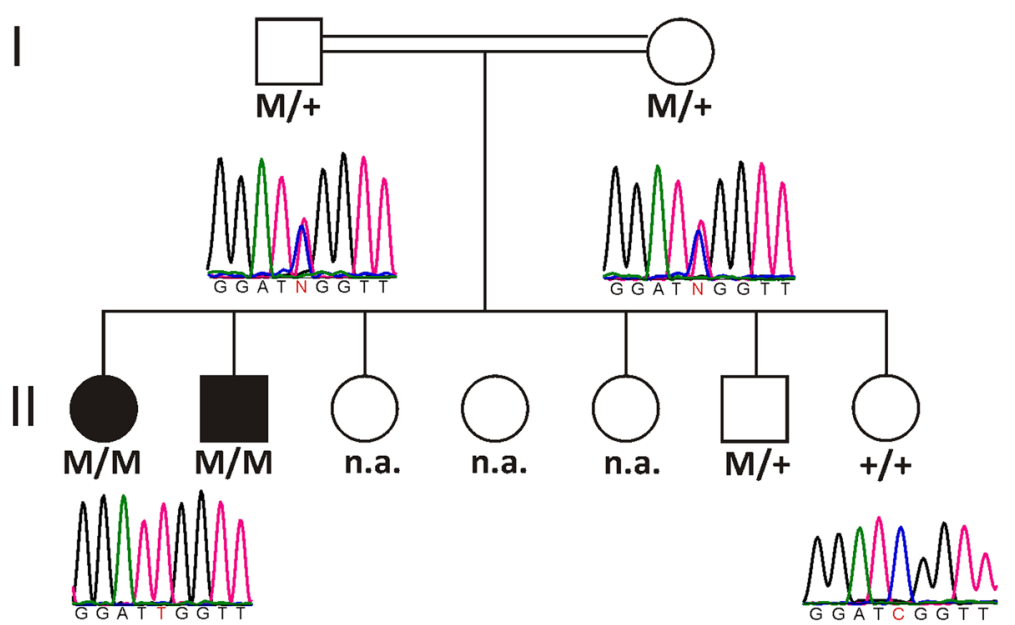

Fig. 1 Pedigrees of families A and B. Genotypes and sequence profiles are given below each available family member. Exonic sequences are given in capital letters and intronic sequences in small letters, respectively. The nucleotides that are changed are highlighted in red. $\mathrm{M}$, mutant allele; +, wildtype allele; n.a., not analyzed

\section{Genetic findings}

DNA of both index patients was analyzed using a panel-based sequencing approach in a diagnostic setting. The gene panel targets 16 genes that are associated with optic atrophy, namely OPA1, OPA3, TMEM126A, WFS1, MFN2, TIMM8A, SPG7, NR2F1, ACO2, RTN4IP1, AFG3L2, C12ORF65, SLC25A46, CISD2, DNM1L, and $Y M E 1 L 1$. No rare and potentially disease-causing exonic variants explaining the disease phenotype were found except for a canonical splice donor site variant in TMEM126A in family $\mathrm{A}$ and a missense variant in the same gene in family B. Both variants segregate with the disease in the families as can be seen in the pedigrees shown in Fig. 1.

The splice donor variant c. $86+2 \mathrm{~T}>\mathrm{C}$ found in family $\mathrm{A}$, which is not present in large population databases 

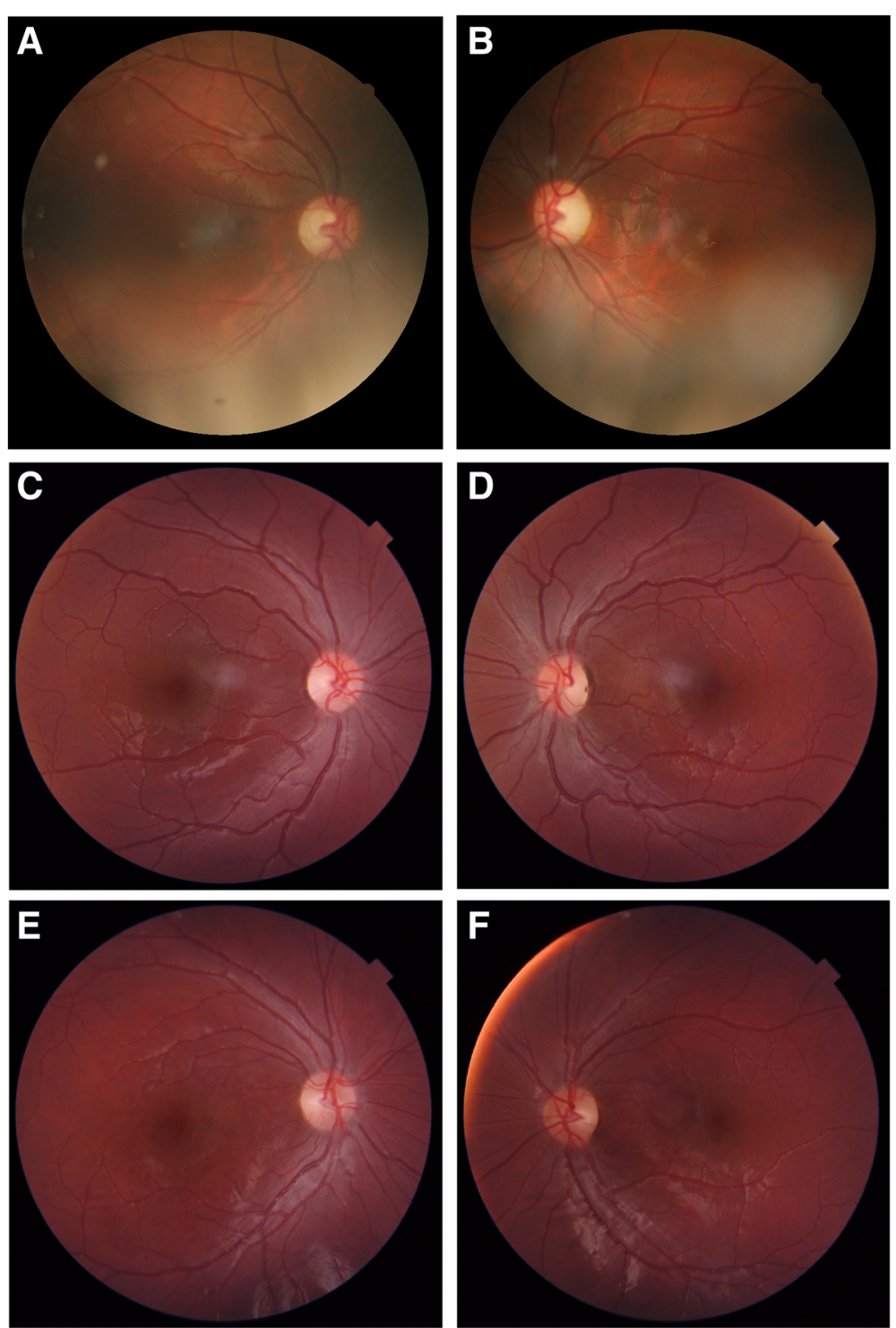

Fig. 2 Eye fundi. Temporal pallor of discs is seen in the right eye (a) and the left eye (b) of the affected proband in family A (ll:1) as well as in the affected sister $(\mathbf{c}+\mathbf{d} ; \|: 1)$ and her brother $(\mathbf{e}+\mathbf{f} ; \|: 2)$ of family $B$

(dbSNP, gnomAD browser), directly changes the invariant $5^{\prime}$ splice core GT sequence of intron 2, ablating the site, with anticipated effects on mRNA splicing. Subsequent cDNA analysis based on RNA isolated from whole blood revealed a single transcript with complete skipping of exon 2 (see Fig. 5). As exon 2 contains the translation start site, the variant c. $86+2 \mathrm{~T}>\mathrm{C}$ presumably results in the failure of translation from its natural start site. There is a shorter isoform of TMEM126A, using an ATG in exon 3, which codes for a protein of 125 aa (Ensembl Transcript ENST00000528105.5). This isoform is observed in normal controls, though the larger isoform (Ensembl Transcript ENST00000304511.6) with the ATG in exon 2 is much more abundant (see Fig. 5a).
This larger isoform is completely lacking in the proband homozygous for the c.86 $+2 \mathrm{~T}>\mathrm{C}$ variant.

The p.(S36 L) variant identified in family B is extremely rare in the general population with a minor allele frequency of 8.122e-6 (sourced from gnomAD). Two different online tools were used to assess the potential pathogenicity of the p.(S36 L) variant, namely the UMD predictor (version March 2018) [22] and the Combined Annotation Dependent Depletion tool (version 1.2) [23]. Within the UMD predictor, scores range from 0 to 100 , with scores $>74$ indicating pathogenicity of a variant. The Combined Annotation Dependent Depletion tool uses a different scoring system with scores $>20$ predicting pathogenicity of a variant. The analysis of the p.(S36 L) variant 

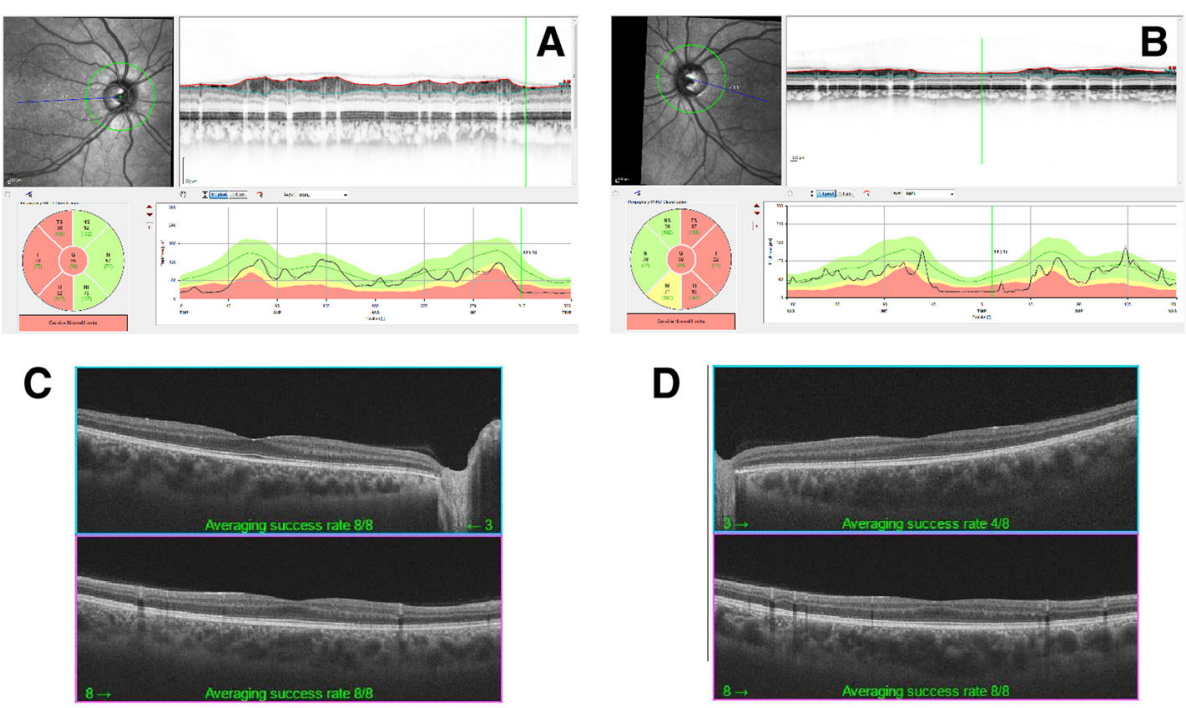

D
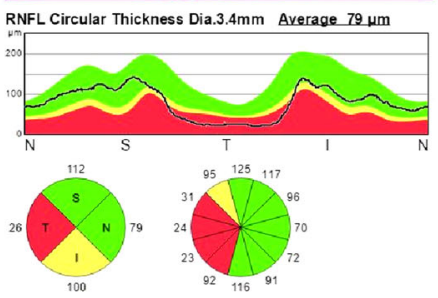

E

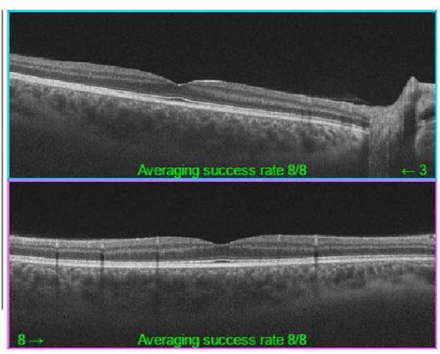

RNFL Circular Thickness Dia.3.4mm Average $89 \mu \mathrm{m}$
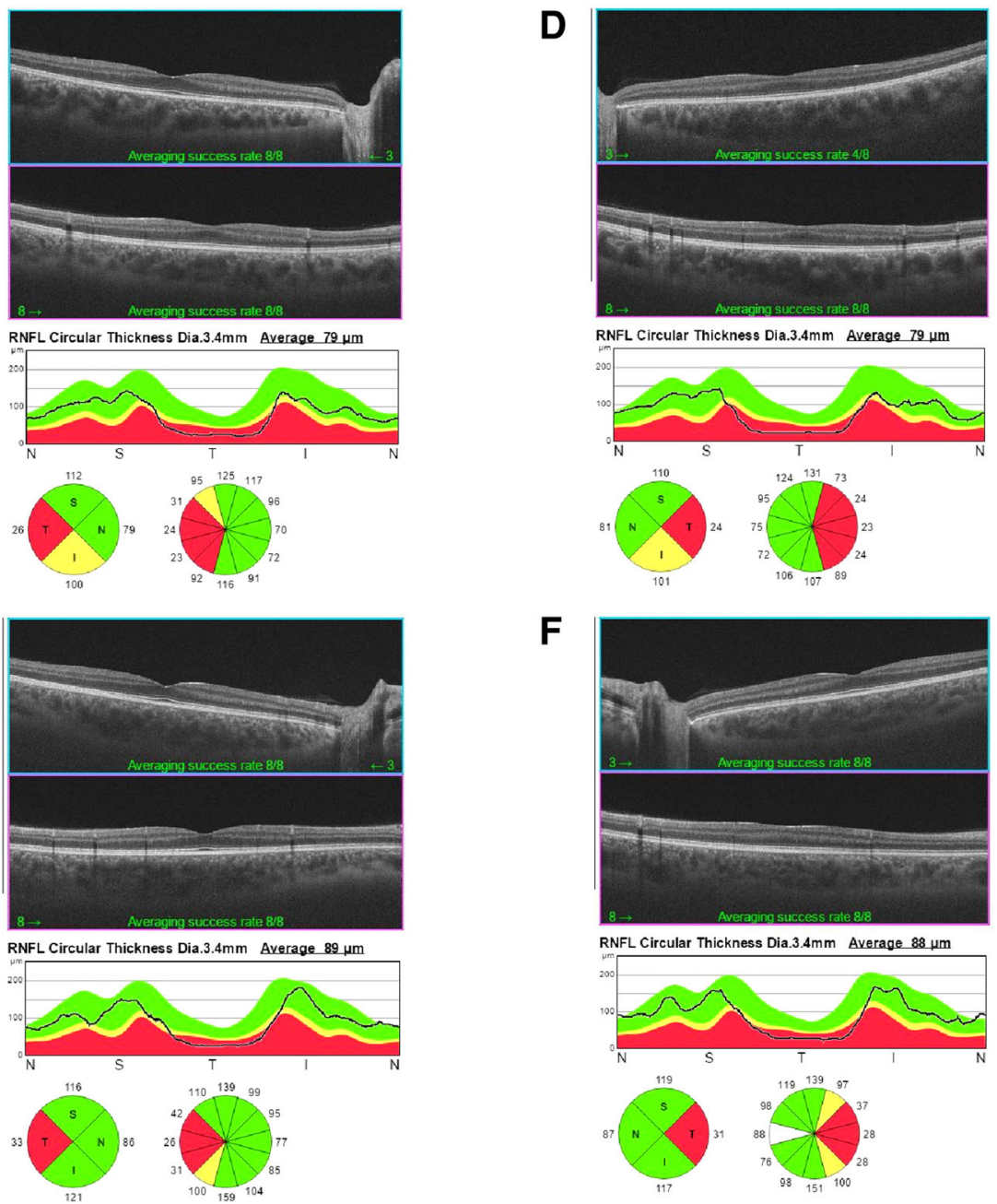

Fig. 3 Optical coherence tomography (OCT) of the retinal nerve fiber layers (RNFLs). (a-b) The right eye (a) and left eye (b) OCT of the affected proband in family A (II:1) show temporal thinning of RNFLs. This can also be seen in the affected sister ( $\mathbf{c}-\mathbf{d}$; $\|: 1)$ and her brother (e-f; $\|: 2)$ of family B

using both tools resulted in scores of 93 and 25, respectively, thereby emphasizing the putative pathogenicity of this novel missense variant.

\section{Discussion and conclusions}

The homozygous nonsense variant (p.R55*) in TMEM126A was proposed as the genetic defect underlying arOA in a large multiplex inbred Algerian family [2]. Clinical features included early-onset severe bilateral deficiency in visual acuity, optic disc pallor, and central scotoma. Subsequently, additional cases, all of Maghrebian descent, carrying the same founder mutation were reported [2-4].

In the current manuscript, we provide further evidence for the implication of biallelic pathogenic TMEM126A variants in arOA, as we diagnosed two additional cases with presumably pathogenic variants in this gene. The index patient of family A, of Turkish descent, carried a novel canonical splice site variant c. $86+2 \mathrm{~T}>\mathrm{C}$, while 


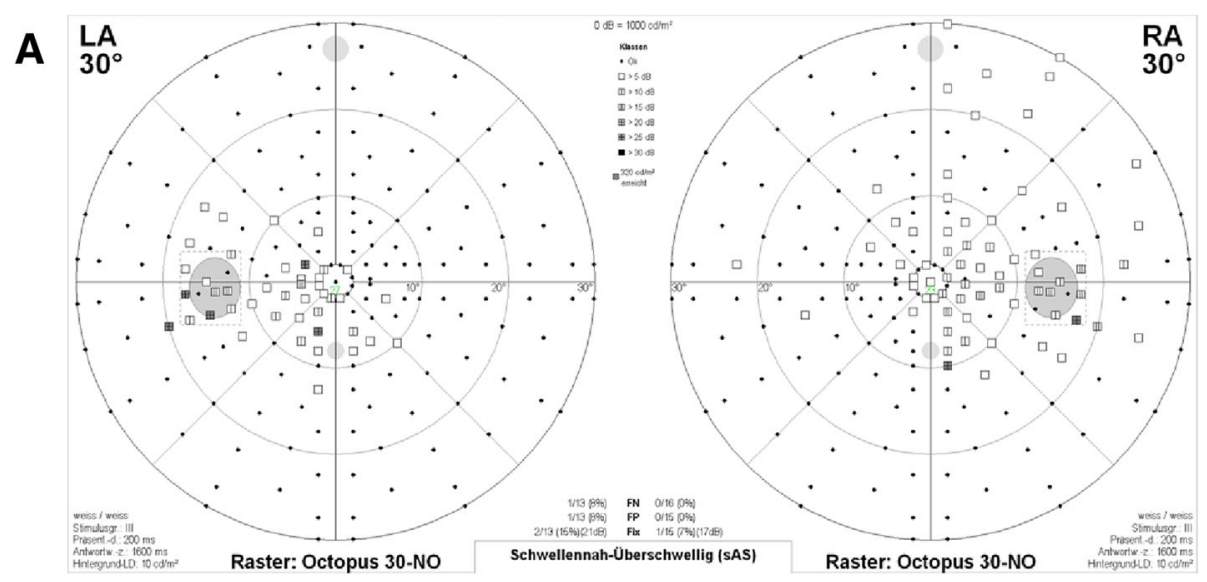

B

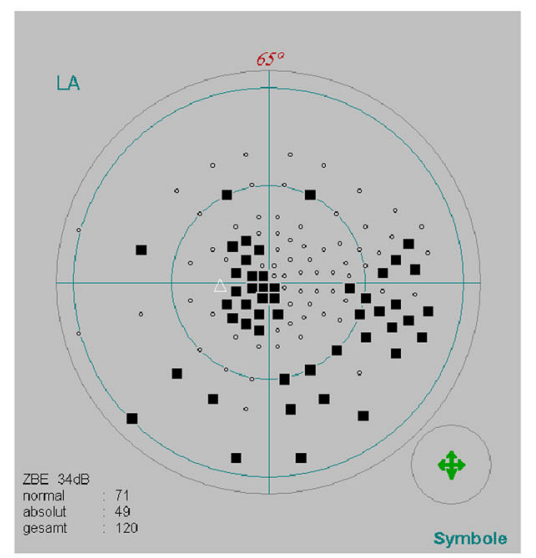

D

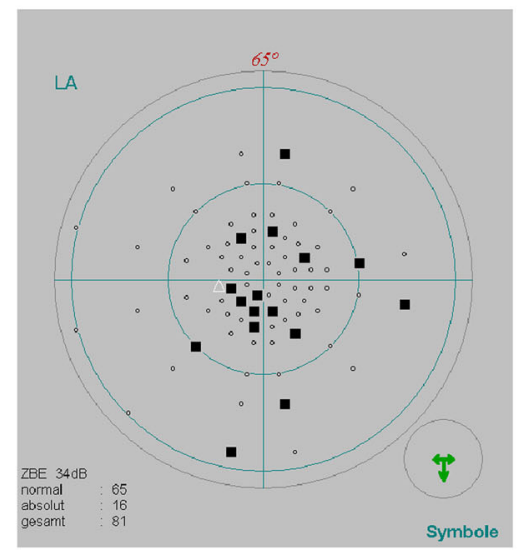

C

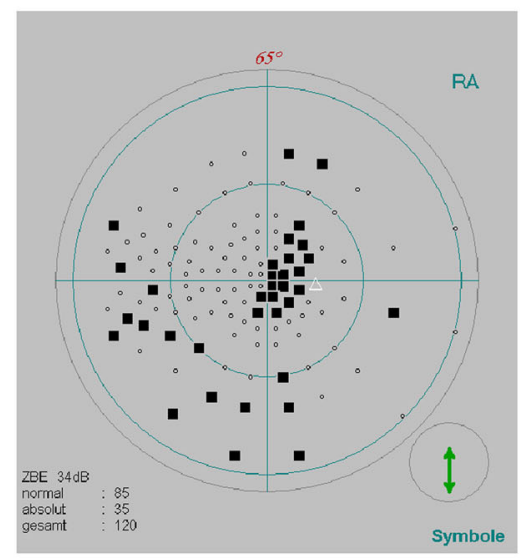

$\mathbf{E}$

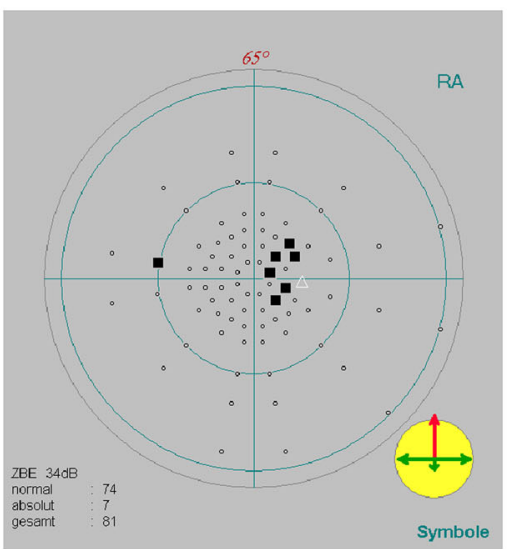

Fig. 4 Perimetry. a The right and left eye visual fields are represented for the affected proband in family A (II:1) and exhibit central scotomas. Scotomas can also be seen in the affected sister (b-c; Il:1) and her brother (d-e; $\|: 2)$ of family B, with the sister showing a more pronounced visual field loss

two affected siblings from family B, of Iraqi descent, carried a novel missense variant, p.(S36 L). Affected members from both families presented with typical symptoms of optic atrophy but revealed no syndromic involvement.

TMEM126A (NP_115649) is predicted to contain four transmembrane (TM) domains with the first being formed by amino acid (aa) residues 39-57 [5]. The function of the
N-terminus (aa 1-38) is completely unknown. One possibility could be that it acts as a mitochondrial targeting leader sequence. However, mitochondrial presequences are usually rich in positively charged aa residues, they have the potential to form amphiphilic alpha-helices and contain consensus motifs for cleavage sites [24], neither of which holds true for the N-terminus of TMEM126A. In fact, it has been shown that it is the second transmembrane 

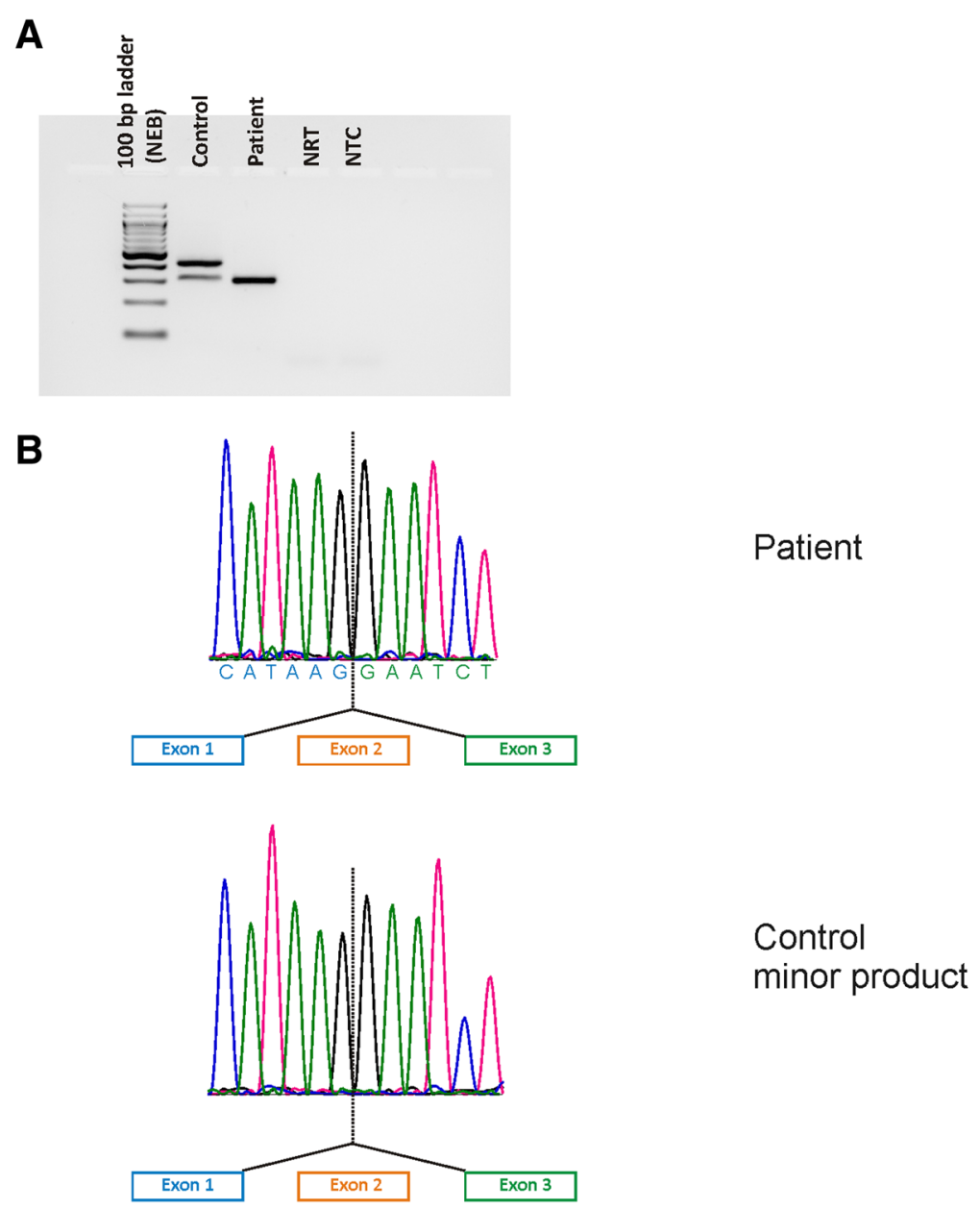

minor product
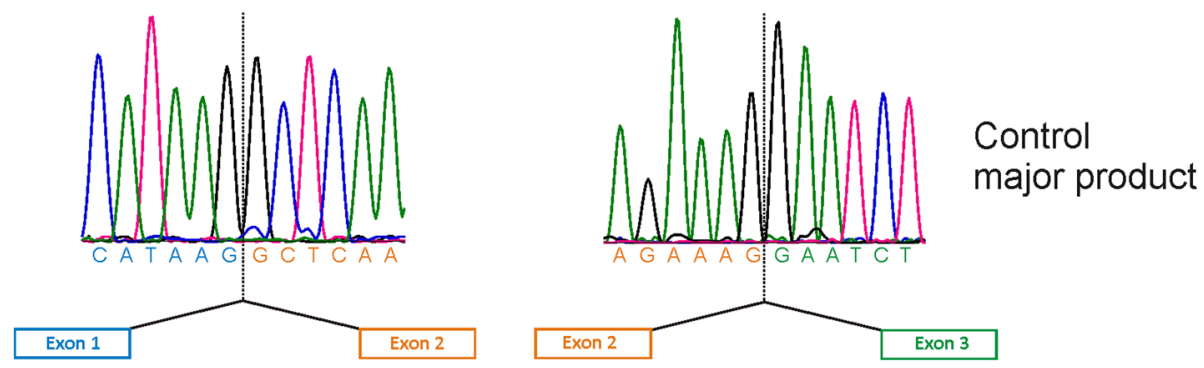

Fig. 5 CDNA analysis of the C.86 $+2 T>C$ variant. a RT-PCR of an unrelated control subject (lane 2) using a forward primer located in exon 1 and a reverse primer in exon 4 revealed an abundant product of $382 \mathrm{bp}$ corresponding to the major isoform of TMEM126A. In addition, a weak product could be identified, which stems from a minor isoform that lacks exon 2. The RT-PCR of the affected proband in family A (II:1) shows that only the minor isoform is present (lane 3). b Sequencing shows skipping of exon 2 in the single RT-PCR product of the affected proband (upper lane) and for the minor RT-PCR product of an unrelated control subject (middle lane) while correct splicing is observed for the abundant RT-PCR product in an unrelated control subject (lower lane). RT-PCR: reverse transcription polymerase chain reaction; NRT, no reverse transcriptase control; NTC, non template control

domain (aa 66-85) that promotes localization to the mitochondria [5]. While it seems obvious that the p.(S36 L) variant does not influence the localization to the mitochondria, it may exert a pathogenic effect through another mechanism. Missense variants may not only act at the protein level but also at the nucleotide level by interfering with the correct assembly of the pre-mRNA splicing machinery. However, in silico analysis of the p.(S36 L) variant using the Human Splicing Finder version 3.0 [25] revealed no difference between mutant and reference sequence. Due to the lack of functional data, and according to the guidelines for the classification of sequence variants by the American 
College of Medical Genetics and Genomics and the Association for Molecular Pathology [26], the p.(S36 L) variant has to be categorized as variant of unclear significance, despite the fact that it represents an ultra-rare variant in the general population, segregates with the disease in the family and affects an evolutionary conserved amino acid residue. On the other hand, we could demonstrate that the canonical splice site variant c.86 $+2 \mathrm{~T}>\mathrm{C}$ leads to the expression of only the short transcript isoform which, if translated, would lack the first seventy amino acids of the canonical protein. This would impair the formation of the second transmembrane domain and we hypothesize that this shortened protein would not be located to the mitochondria.

In conclusion, more information on the effect of TMEM126A alterations on mitochondrial function is needed to confirm the exact pathogenic mechanisms that cause arOA in the probands of this study. Taking our data and the previous publications into account, TMEM126A remains the most promising candidate for non-syndromic arOA and should be considered in affected individuals with a corresponding phenotype (non-syndromic optical atrophy with or without minor extra-ocular findings such as cardiomyopathy, hearing loss, MRI alterations), also in affected individuals outside Maghreb.

\section{Abbreviations \\ aa: Amino acid; adOA: Autosomal-dominant optic atrophy; arOA: Autosomal recessive optic atrophy; LHON: Leber Hereditary Optic Neuropathy; MRI: Magnetic resonance imaging; OCT: Optical coherence tomography; OPA1: Optic atrophy 1; RNFL: Retinal nerve fiber layer; RT-PCR: Reverse transcription polymerase chain reaction; TM: Transmembrane; TMEM126A: Transmembrane protein 126A}

\section{Acknowledgements}

We thank the families for their participation in the study.

\section{Funding}

We acknowledge support by the Deutsche Forschungsgemeinschaft and the Open Access Publishing Fund of the University of Tübingen. Matthis Synofzik is supported by the Else-Kröner Fresenius Stiftung and the grant 779257 "Solve-RD" from the EU Horizon 2020 research and innovation program. The funders had no role in study design, data collection and analysis, decision to publish, or preparation of the manuscript.

\section{Availability of data and materials}

The data that support the findings of this study are available from CeGaT $\mathrm{GmbH}$ and Praxis für Humangenetik Tübingen but restrictions apply to the availability of these data, which were used under license for the current study, and so are not publicly available. Data are however available from the authors upon reasonable request and with permission of $\mathrm{CeGaT} \mathrm{GmbH}$ and Praxis für Humangenetik Tübingen.

\section{Authors' contributions}

KK, CK, MS, AN and FS recruited the families and collected the clinical data. SS-L performed the sequencing data analysis. NW performed the CDNA analysis. BW supervised the study and reviewed the data. NW and KK drafted the manuscript. All authors read and approved the manuscript.

\section{Ethics approval and consent to participate}

Samples from all affected individuals and family members were recruited in accordance with the principles of the Declaration of Helsinki and were obtained with written informed consent accompanying the patients' samples. For the probands of family B who are underage, informed consent for participation in this study was obtained from the probands' parents. The study was approved by the institutional review board of the Ethics Committee of the University Hospital of Tübingen (study number 637/ 2017BO1, dated October 23, 2017) and the Ethics Committee of the Hamburg Chamber of Physicians (study number PV3802, dated March 1, 2016)

\section{Consent for publication}

Both families have given their written consents for the case report to be published. For the probands of family B who are underage, informed consent for publication of this study was obtained from the probands' parents.

\section{Competing interests}

The authors declare that they have no competing interests.

\section{Publisher's Note}

Springer Nature remains neutral with regard to jurisdictional claims in published maps and institutional affiliations.

\section{Author details}

${ }^{1}$ Institute of Human Genetics, University Medical Center Hamburg-Eppendorf, Hamburg, Germany. ${ }^{2}$ Department of Neurodegenerative Diseases, Hertie Institute for Clinical Brain Research, University of Tübingen, Tübingen, Germany. ${ }^{3}$ German Center for Neurodegenerative Diseases (DZNE), Tübingen, Germany. ${ }^{4}$ Center for Ophthalmology, Institute for Ophthalmic Research, University of Tübingen, Tübingen, Germany. ${ }^{5} \mathrm{CeGaT} \mathrm{GmbH}$ and Praxis für Humangenetik Tübingen, Tübingen, Germany. ${ }^{6}$ Department of Ophthalmology, University Medical Center Hamburg-Eppendorf, Hamburg, Germany. ${ }^{7}$ Department of Pediatrics, University Medical Center

Hamburg-Eppendorf, Hamburg, Germany.

Received: 9 October 2018 Accepted: 29 March 2019

Published online: 08 April 2019

References

1. Carelli V, La Morgia C, Ross-Cisneros FN, Sadun AA. Optic neuropathies: the tip of the neurodegeneration iceberg. Hum Mol Genet. 2017;26(R2):R139-50

2. Hanein S, Perrault I, Roche O, Gerber S, Khadom N, Rio M, Boddaert N, JeanPierre M, Brahimi N, Serre V, et al. TMEM126A, encoding a mitochondrial protein, is mutated in autosomal-recessive nonsyndromic optic atrophy. Am J Hum Genet. 2009:84:493-8.

3. Meyer E, Michaelides M, Tee LJ, Robson AG, Rahman F, Pasha S, Luxon LM, Moore AT, Maher ER. Nonsense mutation in TMEM126A causing autosomal recessive optic atrophy and auditory neuropathy. Mol Vis. 2010;16:650-64.

4. Désir J, Coppieters F, Van Regemorter N, De Baere E, Abramowicz M, Cordonnier M. TMEM126A mutation in a Moroccan family with autosomal recessive optic atrophy. Mol Vis. 2012;18:1849-57.

5. Hanein S, Garcia M, Fares-Taie L, Serre V, De Keyzer Y, Delaveau T, Perrault I, Delphin N, Gerber S, Schmitt A, et al. TMEM126A is a mitochondrial located mRNA (MLR) protein of the mitochondrial inner membrane. Biochim Biophys Acta. 2013;1830:3719-33.

6. Delettre C, Lenaers G, Griffoin JM, Gigarel N, Lorenzo C, Belenguer P, Pelloquin L, Grosgeorge J, Turc-Carel C, Perret E, et al. Nuclear gene OPA1, encoding a mitochondrial dynamin-related protein, is mutated in dominant optic atrophy. Nat Genet. 2000;26:207-10.

7. Cipolat S, Martins de Brito O, Dal Zilio B, Scorrano L. OPA1 requires mitofusin 1 to promote mitochondrial fusion. Proc Natl Acad Sci U S A. 2004:101:15927-32.

8. Olichon A, Baricault L, Gas N, Guillou E, Valette A, Belenguer P, Lenaers G. Loss of OPA1 perturbates the mitochondrial inner membrane structure and integrity, leading to cytochrome $\mathrm{c}$ release and apoptosis. J Biol Chem. 2003;278:7743-6.

9. Amati-Bonneau P, Valentino ML, Reynier P, Gallardo ME, Bornstein B, Boissière A, Campos Y, Rivera H, de la Aleja JG, Carroccia R, et al. OPA1 mutations induce mitochondrial DNA instability and optic atrophy 'plus' phenotypes. Brain. 2008;131:338-51.

10. Bonifert T, Karle KN, Tonagel F, Batra M, Wilhelm C, Theurer Y, Schoenfeld C Kluba T, Kamenisch Y, Carelli V, et al. Pure and syndromic optic atrophy explained by deep intronic OPA1 mutations and an intralocus modifier. Brain. 2014;137:2164-77. 
11. Kernstock C, Beisse F, Wiethoff S, Mast A, Krapp E, Grund R, Dietzsch J, Lagrèze W, Fischer D, Schiefer U. Assessment of functional and morphometric endpoints in patients with non-arteritic anterior ischemic optic neuropathy (NAION). Graefes Arch Clin Exp Ophthalmol. 2014;252:515-21.

12. Jiang $H$, Lei R, Ding SW, Zhu S. Skewer: a fast and accurate adapter trimmer for next-generation sequencing paired-end reads. BMC Bioinformatics. 2014;15:182

13. Li H, Durbin R. Fast and accurate short read alignment with burrowswheeler transform. Bioinformatics. 2009;25:1754-60.

14. Sherry ST, Ward MH, Kholodov M, Baker J, Phan L, Smigielski EM, Sirotkin K. dbSNP: the NCBI database of genetic variation. Nucleic Acids Res. 2001;29:308-11.

15. Lek M, Karczewski K, Minikel EV, Samocha KE, Banks E, Fennell T, O'DonnellLuria AH, Ware JS, Hill AJ, Cummings BB, et al. Exome aggregation consortium. Analysis of protein-coding genetic variation in 60,706 humans. Nature. 2016;536:285-91.

16. Liu X, Jian X, Boerwinkle E. dbNSFP: a lightweight database of human nonsynonymous SNPs and their functional predictions. Hum Mutat. 2011;32:894-9.

17. Stenson PD, Mort M, Ball EV, Evans K, Hayden M, Heywood S, Hussain M, Phillips AD, Cooper DN. The human gene mutation database: towards a comprehensive repository of inherited mutation data for medical research, genetic diagnosis and next-generation sequencing studies. Hum Genet. 2017;136:665-77.

18. Aken BL, Achuthan P, Akanni W, Amode MR, Bernsdorff F, Bhai J, Billis K, Carvalho-Silva D, Cummins C, Clapham P, et al. Ensembl 2017. Nucleic Acids Res. 2017:45(D1):D635-42.

19. O'Leary NA, Wright MW, Brister JR, Ciufo S, Haddad D, McVeigh R, Rajput $B$, Robbertse B, Smith-White B, Ako-Adjei D, et al. Reference sequence (RefSeq) database at NCBI: current status, taxonomic expansion, and functional annotation. Nucleic Acids Res. 2016;44(D1):D733-45.

20. Pruitt KD, Harrow J, Harte RA, Wallin C, Diekhans M, Maglott DR, Searle S, Farrell CM, Loveland JE, Ruef BJ, et al. The consensus coding sequence (CCDS) project: identifying a common protein-coding gene set for the human and mouse genomes. Genome Res. 2009;19:1316-23.

21. Weisschuh N, Wissinger B, Gramer E. A splice site mutation in the PAX6 gene which induces exon skipping causes autosomal dominant inherited aniridia. Mol Vis. 2012;18:751-7.

22. Salgado D, Desvignes JP, Rai G, Blanchard A, Miltgen M, Pinard A, Lévy N, Collod-Béroud G, Béroud C. UMD-predictor: a high-throughput sequencing compliant system for pathogenicity prediction of any human CDNA substitution. Hum Mutat. 2016;37:439-46.

23. Kircher M, Witten DM, Jain P, O'Roak BJ, Cooper GM, Shendure J. A genera framework for estimating the relative pathogenicity of human genetic variants. Nat Genet. 2014;46:310-5.

24. Habib SJ, Neupert W, Rapaport D. Analysis and prediction of mitochondria targeting signals. Methods Cell Biol. 2007;80:761-81.

25. Desmet FO, Hamroun D, Lalande M, Collod-Béroud G, Claustres M, Béroud C. Human splicing finder: an online bioinformatics tool to predict splicing signals. Nucleic Acids Res. 2009;37:e67.

26. Richards S, Aziz N, Bale S, Bick D, Das S, Gastier-Foster J, Grody WW, Hegde M, Lyon E, Spector E, et al. ACMG laboratory quality assurance committee. Standards and guidelines for the interpretation of sequence variants: a joint consensus recommendation of the American College of Medical Genetics and Genomics and the Association for Molecular Pathology. Genet Med. 2015;17:405-24.

Ready to submit your research? Choose BMC and benefit from:

- fast, convenient online submission

- thorough peer review by experienced researchers in your field

- rapid publication on acceptance

- support for research data, including large and complex data types

- gold Open Access which fosters wider collaboration and increased citations

- maximum visibility for your research: over $100 \mathrm{M}$ website views per year

At BMC, research is always in progress.

Learn more biomedcentral.com/submissions 